\title{
MOLECULAR LINE OBSERVATIONS OF THE CARBON-RICH CIRCUMSTELLAR ENVELOPE CIT 6 AT 7 mm WAVELENGTHS
}

\author{
Wayne Chau ${ }^{1}$, Yong Zhang (张泳 $)^{1}$, Jun-IChi Nakashima (中岛淳一 $)^{1}$, Shuji Deguchi (出口修至) $)^{2}$, And Sun KwoK (郭新) ${ }^{1}$ \\ ${ }^{1}$ Department of Physics, University of Hong Kong, Hong Kong, China \\ ${ }^{2}$ Nobeyama Radio Observatory, National Astronomical Observatory, Minamimaki, Minamisaku, Nagano 384-1305, Japan \\ Received 2012 July 17; accepted 2012 October 5; published 2012 November 5
}

\begin{abstract}
We present a $\lambda 7 \mathrm{~mm}$ spectral line survey of the carbon-rich circumstellar envelope (CSE) CIT 6 using the $45 \mathrm{~m}$ telescope at the Nobeyama Radio Observatory. A total of 25 spectral features belonging to five molecular species $\left(\mathrm{HC}_{3} \mathrm{~N}, \mathrm{HC}_{5} \mathrm{~N}, \mathrm{HC}_{7} \mathrm{~N}, \mathrm{SiO}\right.$, and $\left.\mathrm{CS}\right)$ are detected, enabling us to investigate the chemistry of cyanopolyyne chains. The line strengths are compared with those of the proto-typical carbon-rich CSE IRC+10216. The results show that the cyanopolyyne molecules are enhanced in CIT 6, suggesting that it is more evolved than IRC+10216. In order to investigate the structure of CIT 6, we have constructed a three-dimensional spatiokinematic model. By comparing the observed line profiles with the models, we conclude that this envelope is asymmetric and is composed of several incomplete shells.
\end{abstract}

Key words: circumstellar matter - ISM: molecules - radio lines: stars - stars: AGB and post-AGB stars: individual (CIT 6)

Online-only material: color figures

\section{INTRODUCTION}

Facilitated by the dredge-up of carbon from the stellar interior to the surface, the circumstellar envelopes (CSEs) of carbonrich asymptotic giant branch (AGB) stars have been found to be active sites of formation of carbon-based molecules (see, e.g., Kwok 2004, 2009; Ziurys et al. 2007). In the last stage of stellar evolution, beginning with AGB stars evolving to protoplanetary nebulae (PPNe) and then to planetary nebulae (PNe), the physical conditions, such as density, temperature, and radiation field, in the CSE are rapidly changing as the result of the evolution of the central star and mass loss. These environmental changes lead to the initiation of different chemical reactions, resulting in a rich variety of molecular species. Identification and analysis of molecular emission lines in objects at different stages of stellar evolution can therefore facilitate the understanding of circumstellar chemistry (see Cernicharo et al. 2011 for a recent review). However, such studies have hitherto only focused on a few bright evolved stars or a small number of molecular species. For this reason, we have been performing a long-term project of systematic spectral line surveys of a large sample of evolved stars at wide wavelength ranges (He et al. 2008; Zhang et al. 2008, 2009a, 2009b). The present paper is the fifth one of this series.

Because of its proximity to us, IRC +10216 (CW Leo) is the most surveyed C-rich AGB star. With over 70 molecular species detected, IRC+10216 is one of the richest sources of molecular lines in the sky (see, e.g., He et al. 2008; Cernicharo et al. 2000, 2010; Patel et al. 2011, etc.), and it is often considered as a prototype for late-type carbon stars. However, a crucial question is whether IRC+10216 is chemically similar to other evolved stars so that it is appropriate to take it as a reference to study circumstellar chemistry. To investigate this problem, more carbon stars must be surveyed at wider wavelength ranges. In our previous works (Zhang et al. 2009a, 2009b), we have obtained the $\lambda 1.3 \mathrm{~mm}$ and $2 \mathrm{~mm}$ spectra of CIT 6 and CRL 3068 using the Arizona Radio Observatory's $12 \mathrm{~m}$ and $10 \mathrm{~m}$ Submillimeter Telescopes, and found that the chemical compositions of both sources are similar to that of IRC+10216. More evolved carbon stars that have been studied through line surveys include the PPN CRL 2688 (Park et al. 2008), the PPN CRL 618 (Pardo et al. 2007), and the young PN NGC 7027 (Zhang et al. 2008). Studying these objects, which are in consecutive stages of evolution, provides useful information on molecular chemical synthesis and evolution.

Beside stellar evolution, the $\mathrm{C} / \mathrm{O}$ ratio is another important factor determining the chemical composition of CSEs. Line surveys of objects with different $\mathrm{C} / \mathrm{O}$ ratio enable us to understand the chemical processes in different environments. For example, Zhang et al. (2009b) found that the extremely carbon-rich environment of CRL 3068 favors the production of cyclic and long-chain carbon molecules. Ziurys et al. (2007) demonstrated the chemical complexity of oxygen-rich CSEs. The first line survey of an oxygen-rich supergiant, VY Canis Majoris, was presented by Tenenbaum et al. (2010) and was compared to that of IRC+10216.

As the second brightest C-rich star after IRC+10216, CIT 6 is an excellent target for line survey. The properties of CIT 6 have been outlined extensively in Woods et al. (2003b). Similar to IRC+10216, CIT 6 is a long-period variable with a period of $~ 640$ days, with which Cohen \& Hitchon (1996) derived a distance of $\sim 400 \pm 50 \mathrm{pc}$. The structure of CIT 6 is also intriguing. Optical images show that the central region has a bipolar structure (Schmidt et al. 2002), while the molecular line maps of Lindqvist et al. (2000) indicate a large-scale asymmetry in the CSE. Based on $\mathrm{HC}_{3} \mathrm{~N}$ observations, Dinh \& Lim (2009) hypothesized that CIT 6 has a spiral structure that may originate from the interaction of a binary system. However, a more recent observation with improved spatial resolution by Claussen et al. (2011) suggests that the envelope is composed of a series of incomplete shells.

In this paper, we extend our previous work (Zhang et al. 2009a, hereafter Z09) on the line survey for CIT 6 to $\lambda 7 \mathrm{~mm}$ wavelengths. This allows the detection of lines belonging to larger cyanopolyyne molecules. The paper is organized as follows. Section 2 provides a brief account of the observations. 


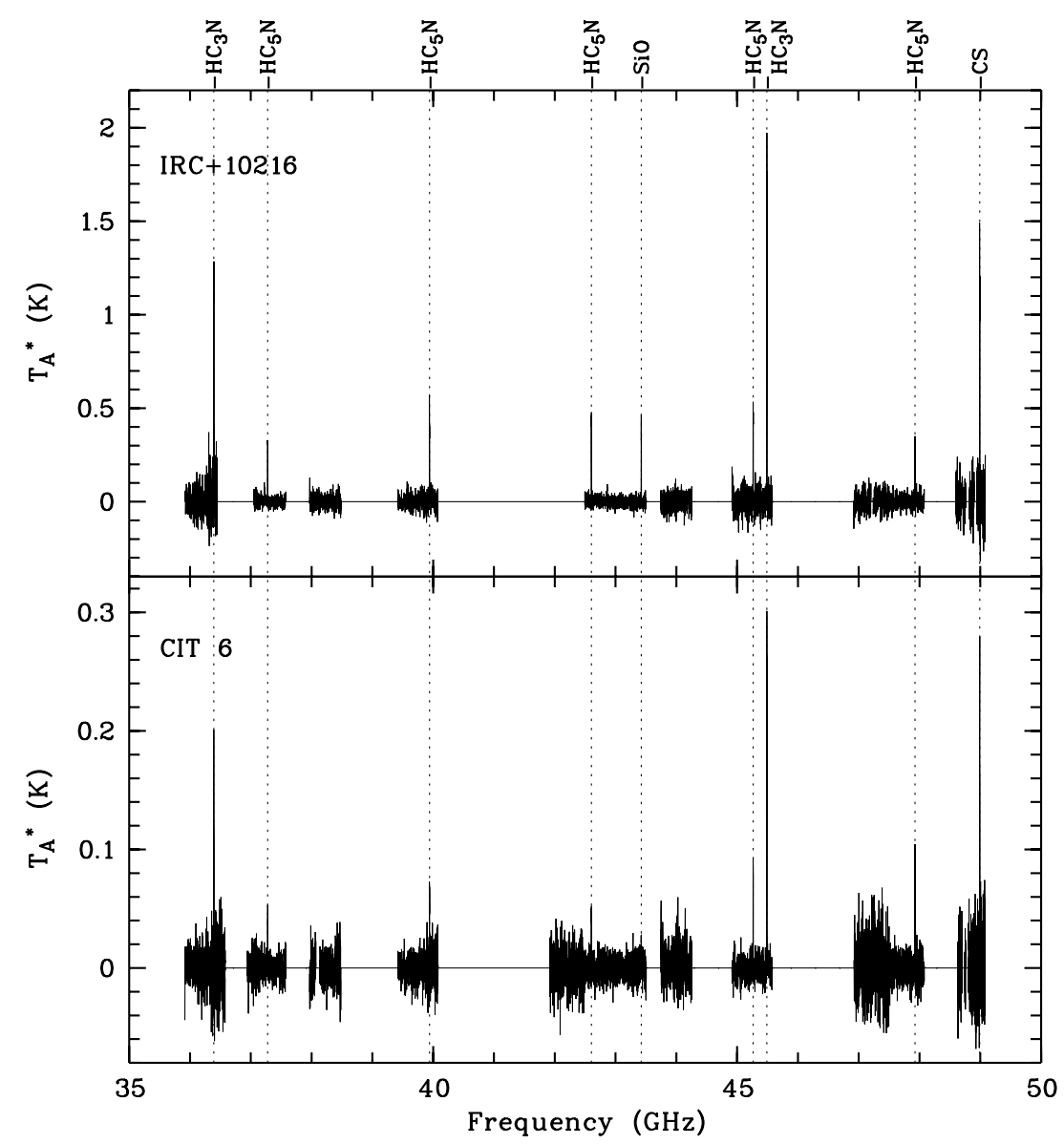

Figure 1. $\lambda 7 \mathrm{~mm}$ spectrum of IRC+10216 (top) and CIT 6 (bottom). Identified molecular lines have been indicated accordingly.

Section 3 lays out the identification of the emission lines and the calculation of the excitation temperatures as well as the fractional abundances. The implications of our observations on circumstellar chemistry and structure of the envelope are discussed in Section 4. The conclusions are given in Section 5.

\section{OBSERVATION AND DATA REDUCTION}

The $\lambda 7 \mathrm{~mm}$ observations of CIT 6 were made using the SIS receiver at the Nobeyama $45 \mathrm{~m}$ radio telescope in 2010 March. The receiver has a band width of $650-700 \mathrm{MHz}$ and a system temperature of 150-200 K (see Kawaguchi et al. 1995 for details of the instrument). The observations were made in the positionswitching mode, with on-source integration time of about $2 \mathrm{hr}$ for each frequency setting. The telescope pointing was checked every $2 \mathrm{hr}$ by observing bright $\mathrm{SiO}$ maser sources, for which another receiver covering a frequency range from 42 to $44 \mathrm{GHz}$ was used by switching a mirror. The frequency range of the target observations was from 36 to $49 \mathrm{GHz}$ with some gaps (Figure 1). We have taken the spectra of IRC+10216 obtained by Kawaguchi et al. (1995) as a reference and preferentially observed the frequency settings covering strong lines. Under the assumption that all the lines in CIT 6 are 10 times fainter than those in IRC+10216 (see below for the validity of this assumption), it is expected that all the molecular lines within the unobserved frequency ranges are well below the detection limit.

The NewStar ${ }^{3}$ software package is used for the data reduction. A low-order polynomial was used to fit the baseline. The

\footnotetext{
3 http://www.nro.nao.ac.jp/ nro45mrt/obs/newstar
}

co-adding spectra have a typical rms noise temperature of less than $20 \mathrm{mK}$ in antenna temperature $\left(T_{A}^{*}\right)$. Figure 2 shows the zoomed-in spectra along with the rms noise of each setting. For the purpose of comparison and examining the reliability of calibrations and measurements, we also took short-integration ( $\sim 15$ minutes) spectra of IRC+10216 in the same settings with CIT 6, as shown in Figure 1. Since both CIT 6 and IRC+10216 are observed within the same period in this survey, the calibration errors introduced by instrumental variation and weather have been greatly reduced, thereby providing a more accurate comparison between the two objects.

\section{RESULTS}

\subsection{Detection of Molecular Lines}

This radio survey resulted in the positive detection of 14 rotational transition lines belonging to five molecular species in CIT 6 and 11 lines belonging to the same five species in IRC+10216. Listed in Table 1 are the central rest frequencies of the detected lines with their assignments as well as the peak and integrated intensities for both objects. The full width at zero intensity (FWZI) values are also provided for reference. In the presence of unresolved hyperfine structures, both the integrated intensity and FWZI are those of the combined profile. Values from Kawaguchi et al. (1995) are also included for comparison. Uncertainties in the integrated intensities are approximated by the product of the FWZI and rms noise. With the exception of the line corresponding to the $J=1-0$ transition of CS, the integrated intensities of IRC+10216 measured in this survey 

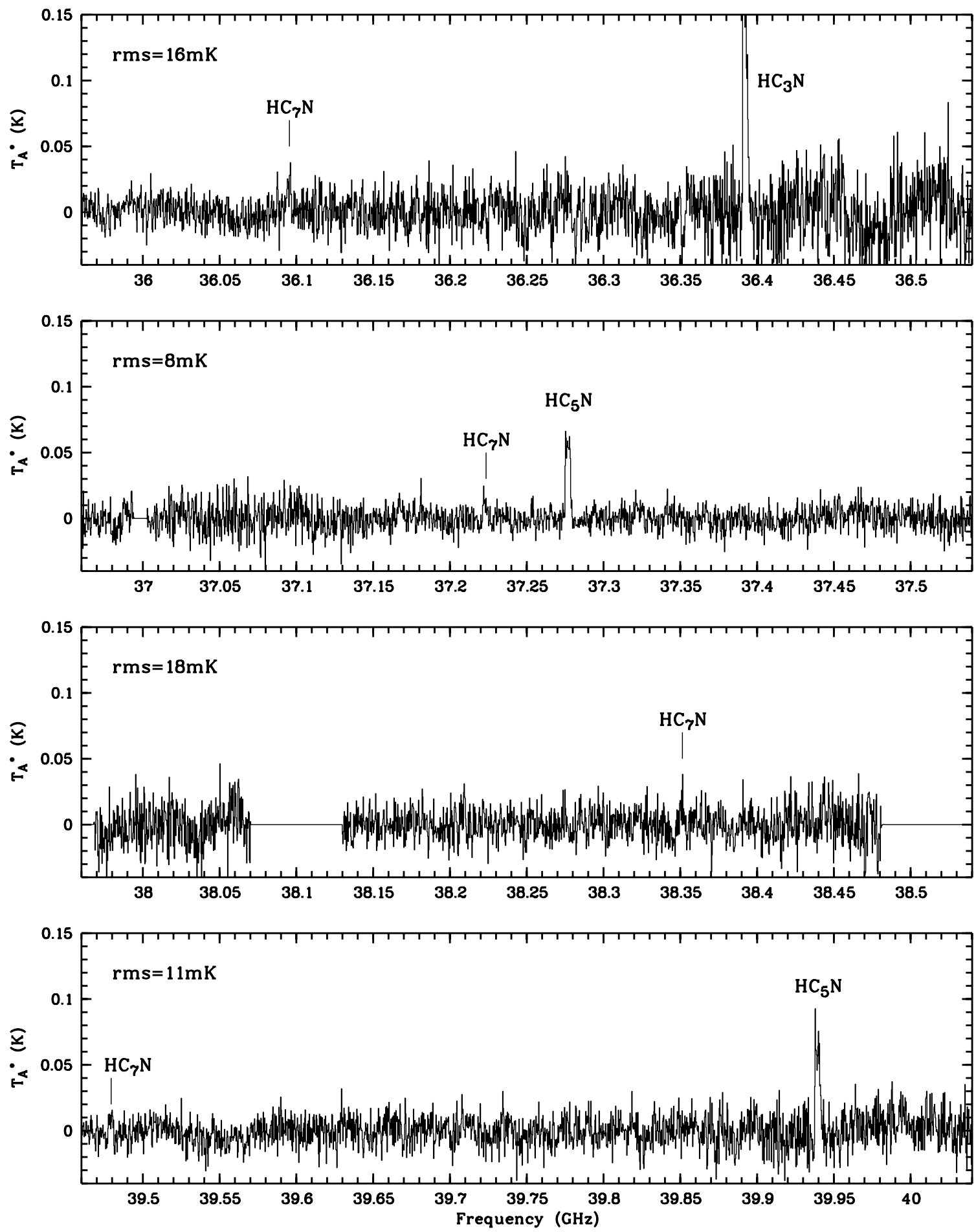

Figure 2. Zoom-in view of the spectrum of CIT 6. The rms is indicated for each wavelength range.

agree well with those obtained by Kawaguchi et al. (1995). Described below are the details of each detected molecule in this $\lambda 7 \mathrm{~mm}$ survey for CIT 6 and IRC+10216.

The $J=4-3$ and $J=5-4$ transitions for $\mathrm{HC}_{3} \mathrm{~N}$ were detected in both objects. In CIT 6, as shown in Figure 3, both lines exhibit a brighter red wing with a sharp low-frequency dropoff. The high-frequency drop-off, by contrast, is much more gentle. This behavior is also observed in Dinh \& Lim (2009) and the higher- $J$ transitions in Z09. The trend is less apparent in IRC+10216. From the interferometric measurements of the
$J=4-3$ transition in Claussen et al. (2011), the diameter of the emission region of $\mathrm{HC}_{3} \mathrm{~N}$ is about 20 arcsec.

Emission lines belonging to five rotational transitions of $\mathrm{HC}_{5} \mathrm{~N}$ are also clearly detected in both objects. Transitions $J=18-17$ and $J=17-16$ in CIT 6 display a brighter red wing; however, this characteristic is much less apparent in the lower-order transitions detected and in all $\mathrm{HC}_{5} \mathrm{~N}$ profiles of IRC+10216. The line profiles in both objects also have a double peak with variable degree of asymmetry, suggesting that the lines are likely to be optically thin. $\mathrm{As} \mathrm{HC}_{5} \mathrm{~N}$ is a daughter 

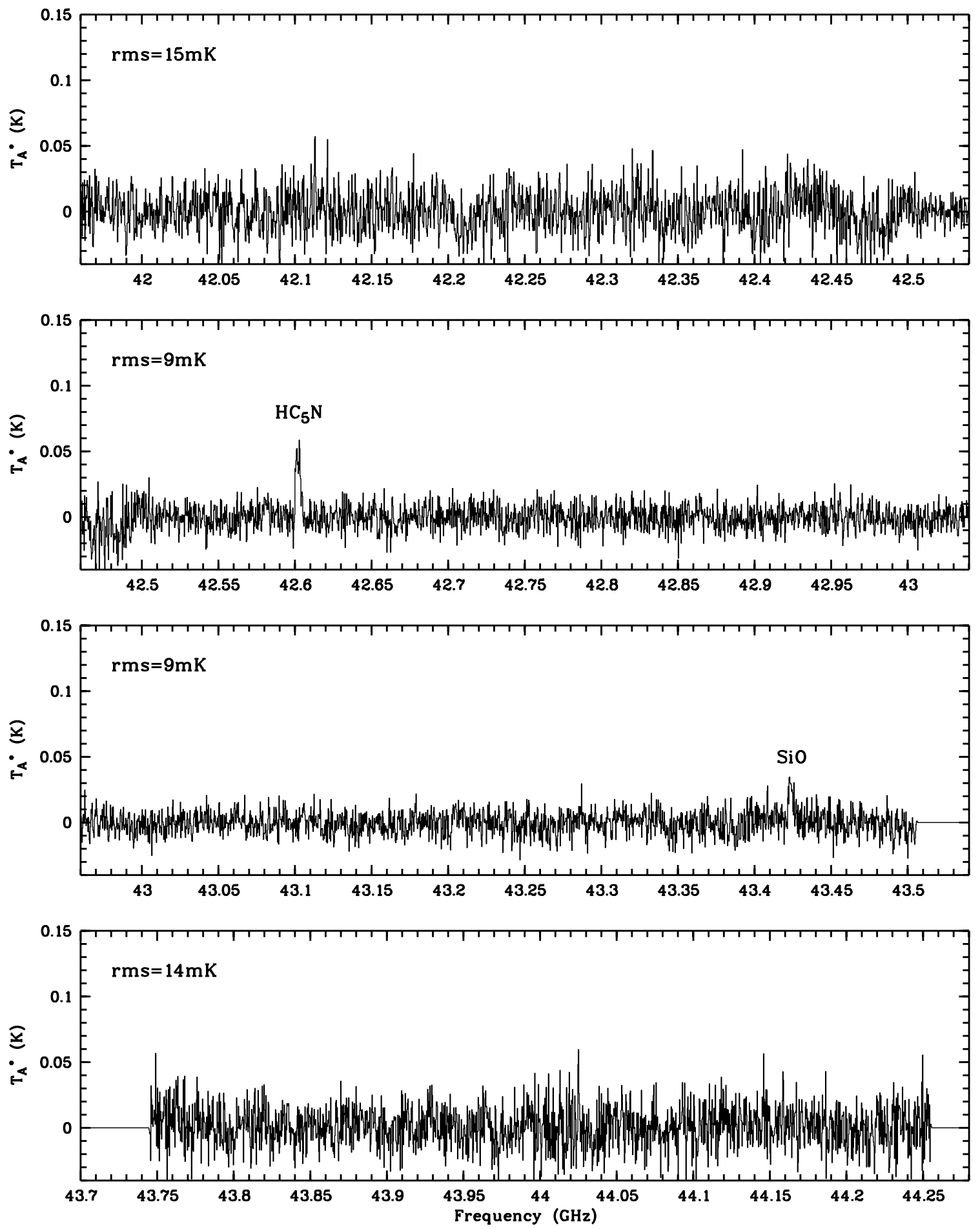

Figure 2. (Continued)

molecule of $\mathrm{HC}_{3} \mathrm{~N}$, the size of its emission region can be approximated by that of $\mathrm{HC}_{3} \mathrm{~N}$.

Five $\mathrm{HC}_{7} \mathrm{~N}$ lines are positively identified in CIT 6 (see Figure 2). To our knowledge, these are new detections. The faintness of these lines makes it difficult to describe their profiles in detail as they are close to the order of the rms noise. The emission region of $\mathrm{HC}_{7} \mathrm{~N}$ should also be similar to that of its parent molecules $\mathrm{HC}_{3} \mathrm{~N}$ and $\mathrm{HC}_{5} \mathrm{~N}$.

The $J=1-0$ line of $\mathrm{SiO}$ is extremely faint in CIT 6 and is the only one from an oxygen-bearing molecule in this survey.
Due to its faintness, features on the line profile are barely identifiable. From the mass-loss rate and expansion velocity, Schöier et al. (2006) calculated the diameter of the $\mathrm{SiO}$ envelope to be 2.6 arcsec, which is significantly smaller than those of the cyanopolyyne molecules.

The $J=1-0 \mathrm{CS}$ line is the second strongest one detected in this survey. Its profile shows slightly asymmetry. Kawaguchi et al. (1995) obtained the intensity of the CS line to be about two times greater than our value for IRC+10216. Although this might be due to uncertainties in the calibration of system 

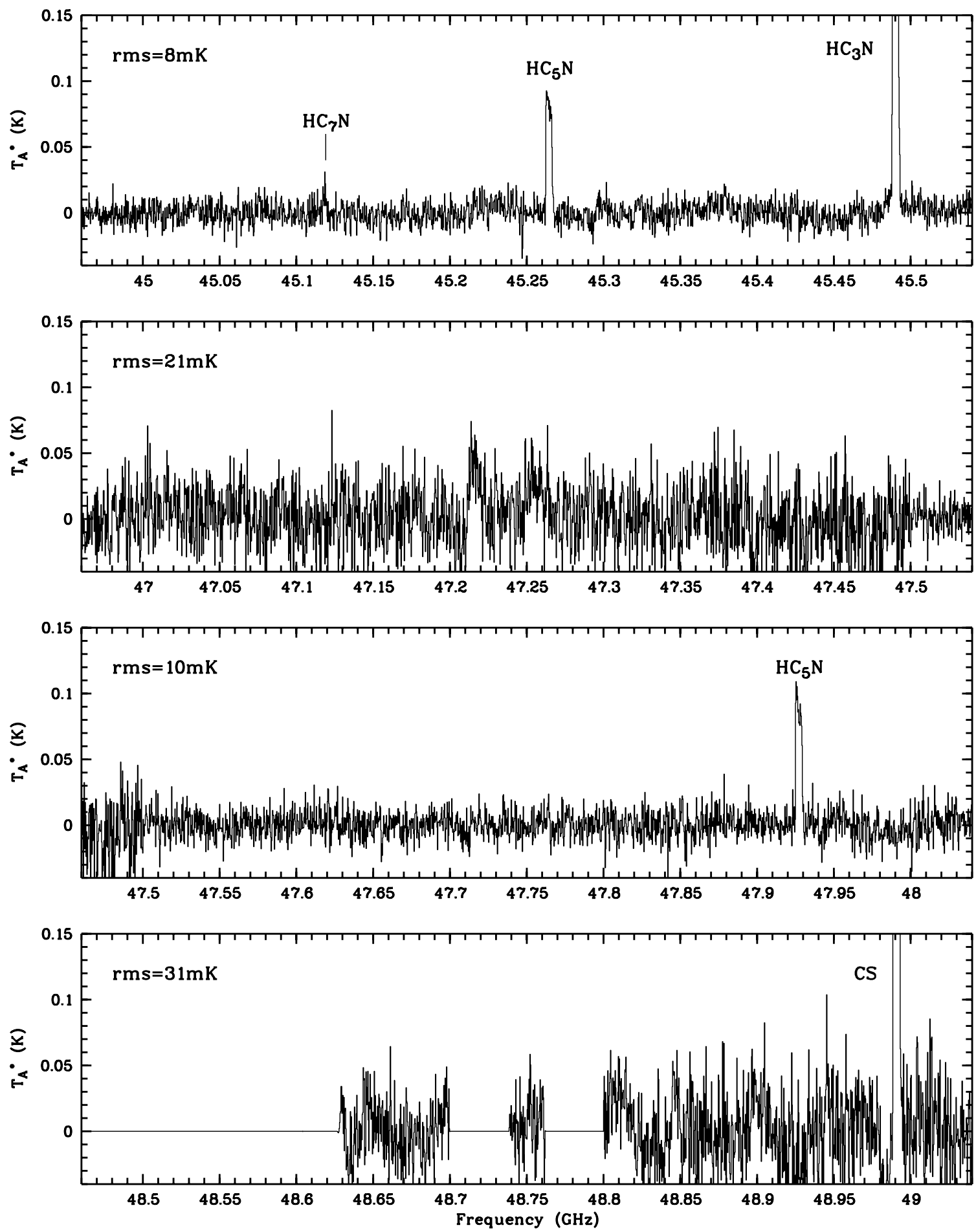

Figure 2. (Continued)

temperature and pointing, we cannot rule out the possibility that the strength of CS lines changes with time since IRC+10216 is variable.

\subsection{Rotational Analysis and Fractional Abundances}

The excitation temperatures and column densities of detected molecules are estimated using rotational diagrams, which are plots of the natural log of the upper level population against its excitation energy. Under the assumption of optically thin emission and local thermal equilibrium (LTE), the level populations are calculated from the integrated intensity following

$$
\ln \frac{N_{u}}{g_{u}}=\ln \frac{3 k \int T_{S} d v}{8 \pi^{3} v \mu^{2} S}=\ln \frac{N}{Q}-\frac{E_{u}}{k T_{\mathrm{ex}}},
$$

where $\mu^{2} S$ is the square of the dipole moment multiplied by the line strength, $g_{u}$ is the degeneracy of the upper level, and $Q$ is the partition function. With the integrated intensity, $\int T_{S} d v$, obtained from the observational data, one can plot the left-hand side of Equation (1) against $E_{u} / k$ to create rotational diagrams. The excitation temperature is then the inverse of the slope of the 
Table 1

Molecular Transitions Detected in CIT 6 and IRC+10216

\begin{tabular}{|c|c|c|c|c|c|c|c|c|c|c|}
\hline \multirow{2}{*}{$\begin{array}{l}\text { Frequency } \\
(\mathrm{MHz})\end{array}$} & \multirow[t]{2}{*}{ Species } & \multirow{2}{*}{$\begin{array}{c}\text { Transition } \\
\text { (Upper-lower) }\end{array}$} & \multicolumn{4}{|c|}{ CIT 6} & \multicolumn{4}{|c|}{$\mathrm{IRC}+10216^{\mathrm{a}}$} \\
\hline & & & $\begin{array}{c}\mathrm{rms} \\
(\mathrm{mK})\end{array}$ & $\begin{array}{c}T_{A}^{*} \\
(\mathrm{~K})\end{array}$ & $\underset{\left(\mathrm{K} \mathrm{km} \mathrm{s}^{-1}\right)}{\int T_{A}^{*} \mathrm{~d} v}$ & $\begin{array}{c}\Delta v_{\mathrm{FWZI}} \\
\left(\mathrm{K} \mathrm{km} \mathrm{s}^{-1}\right)\end{array}$ & $\begin{array}{l}\mathrm{rms} \\
(\mathrm{mK})\end{array}$ & $\begin{array}{c}T_{A}^{*} \\
(\mathrm{~K})\end{array}$ & $\begin{array}{c}\int T_{A}^{*} \mathrm{~d} v \\
\left(\mathrm{~K}\left(\mathrm{~K} \mathrm{~km} \mathrm{~s}^{-1}\right)\right.\end{array}$ & $\begin{array}{c}\Delta v_{\mathrm{FWZI}} \\
\left(\left(\mathrm{K} \mathrm{km} \mathrm{s}^{-1}\right)\right.\end{array}$ \\
\hline 36392.3 & $\mathrm{HC}_{3} \mathrm{~N}$ & $4-3$ & 16 & 0.201 & $4.56 \pm 0.70$ & 43.8 & 155 & $1.285 / 1.351$ & $32.87 \pm 5.47 / 36.58$ & 35.4 \\
\hline 45490.3 & $\mathrm{HC}_{3} \mathrm{~N}$ & $5-4$ & 8 & 0.329 & $7.76 \pm 0.31$ & 38.4 & 61 & $1.963 / 2.403$ & $52.60 \pm 2.24 / 61.33$ & 36.7 \\
\hline 37277.0 & $\mathrm{HC}_{5} \mathrm{~N}$ & $14-13$ & 8 & 0.065 & $1.67 \pm 0.31$ & 38.5 & 17 & $0.326 / 0.400$ & $8.73 \pm 0.61 / 10.82$ & 36.1 \\
\hline 39939.6 & $\mathrm{HC}_{5} \mathrm{~N}$ & $15-14$ & 11 & 0.091 & $1.70 \pm 0.38$ & 34.3 & 35 & $0.571 / 0.379$ & $13.06 \pm 1.06 / 10.72$ & 30.6 \\
\hline 42602.2 & $\mathrm{HC}_{5} \mathrm{~N}$ & $16-15$ & 9 & 0.059 & $1.33 \pm 0.31$ & 36.4 & 25 & $0.480 / 0.464$ & $13.07 \pm 0.76 / 11.87$ & 30.9 \\
\hline 45264.7 & $\mathrm{HC}_{5} \mathrm{~N}$ & $17-16$ & 8 & 0.091 & $2.39 \pm 0.28$ & 34.8 & 42 & $0.529 / 0.604$ & $13.61 \pm 1.39 / 15.58$ & 33.6 \\
\hline 47927.3 & $\mathrm{HC}_{5} \mathrm{~N}$ & $18-17$ & 10 & 0.108 & $2.55 \pm 0.36$ & 35.6 & 31 & $0.347 / 0.566$ & $10.78 \pm 0.94 / 14.46$ & 30.6 \\
\hline 36095.5 & $\mathrm{HC}_{7} \mathrm{~N}$ & $32-31$ & 16 & 0.036 & $0.54 \pm 0.53$ & 33.1 & $\ldots$ & $\ldots / 0.083$ & $\ldots / 2.48$ & $\ldots$ \\
\hline 37223.5 & $\mathrm{HC}_{7} \mathrm{~N}$ & $33-32$ & 8 & 0.014 & $0.50:$ & $\ldots$ & 21 & $0.095 / 0.092$ & $2.47 \pm 0.67 / 2.48$ & 31.5 \\
\hline 38351.4 & $\mathrm{HC}_{7} \mathrm{~N}$ & $34-33$ & 18 & 0.019 & $0.55:$ & $\ldots$ & 45 & $0.088 / 0.093$ & $2.95 \pm 1.64 / 2.10$ & 36.5 \\
\hline 39479.4 & $\mathrm{HC}_{7} \mathrm{~N}$ & $35-34$ & 11 & 0.016 & $0.30:$ & $\ldots$ & $\ldots$ & $\ldots / 0.097$ & $\ldots / 2.56$ & $\ldots$ \\
\hline 45119.1 & $\mathrm{HC}_{7} \mathrm{~N}$ & $40-39$ & 8 & 0.029 & $0.25:$ & $\ldots$ & $\ldots$ & $\ldots / 0.103$ & $\ldots / 2.58$ & $\ldots$ \\
\hline 43423.8 & $\mathrm{SiO}$ & $1-0$ & 9 & 0.035 & $0.62 \pm 0.29$ & 31.7 & 20 & $0.466 / 0.462$ & $11.62 \pm 0.72 / 11.62$ & 35.2 \\
\hline 48991.0 & $\mathrm{CS}$ & $1-0$ & 31 & 0.302 & $6.75 \pm 1.10$ & 35.5 & 139 & $1.488 / 2.654$ & $34.37 \pm 4.46 / 61.46$ & 32.2 \\
\hline
\end{tabular}

Note. ${ }^{a}$ This paper/Kawaguchi et al. (1995).

Table 2

Excitation Temperature and Column Density of Identified Cyanopolyyne Molecules Obtained from Rotational Analysis for CIT 6 and IRC+10216

\begin{tabular}{|c|c|c|c|c|c|c|c|c|}
\hline \multirow[t]{2}{*}{ Species } & \multicolumn{4}{|c|}{ CIT 6} & \multicolumn{4}{|c|}{ IRC+10216 } \\
\hline & $\begin{array}{l}T_{\mathrm{ex}} \\
(\mathrm{K})\end{array}$ & $\begin{array}{c}\delta_{T_{\mathrm{ex}}} \\
( \pm \mathrm{K})\end{array}$ & $\begin{array}{c}N \\
\left(10^{14} \mathrm{~cm}^{-2}\right)\end{array}$ & $\begin{array}{c}\delta_{N} \\
\left( \pm 10^{14} \mathrm{~cm}^{-2}\right)\end{array}$ & $\begin{array}{l}T_{\mathrm{ex}} \\
(\mathrm{K})\end{array}$ & $\begin{array}{c}\delta_{T_{\mathrm{ex}}} \\
( \pm \mathrm{K})\end{array}$ & $\begin{array}{c}N \\
\left(10^{14} \mathrm{~cm}^{-2}\right)\end{array}$ & $\begin{array}{c}\delta_{N} \\
\left( \pm 10^{14} \mathrm{~cm}^{-2}\right)\end{array}$ \\
\hline $\mathrm{HC}_{3} \mathrm{~N}$ & $11.8^{\mathrm{a}}$ & $0.5^{\mathrm{a}}$ & 3.3 & 0.7 & 6.1 & $\ldots$ & 23 & 6 \\
\hline $\mathrm{HC}_{5} \mathrm{~N}$ & 20 & 10 & 1.3 & 0.2 & 11 & 3 & 8.1 & 0.2 \\
\hline $\mathrm{HC}_{7} \mathrm{~N}$ & 9 & 1 & 0.8 & 0.3 & $\ldots$ & $\ldots$ & 5.0 & 0.6 \\
\hline
\end{tabular}

Note. ${ }^{a}$ Concatenated with data from Zhang et al. (2009a).

linear fit for such a plot. The source brightness temperature $T_{S}$ is related to the antenna temperature $T_{A}^{*}$ by

$$
T_{S}=\frac{1}{\eta} T_{R}=\frac{1}{\eta} \frac{\theta_{b}^{2}+\theta_{S}^{2}}{\theta_{S}^{2}} T_{A}^{*}
$$

where $\eta$ is the main beam efficiency, $\theta_{b}$ is the half-power beam width (HPBW $=34 . .9-41^{\prime \prime} .5$ for the observed frequency range) of the Gaussian beam, and $\theta_{S}$ is the angular size of the source, which is, in general, different for different molecular species. The factor $\left(\theta_{b}^{2}+\theta_{S}^{2}\right) / \theta_{S}^{2}$ is introduced to account for beam dilution. For the Nobeyama data, $\eta$ is taken to be 0.87 , while $\theta_{b}$, a frequency-dependent quantity, is found by linear extrapolation of the values in Kawaguchi et al. (1995). The resulting excitation temperatures and column densities are given in Table 2.

The rotational diagram for $\mathrm{HC}_{3} \mathrm{~N}$ is shown in Figure 4, where data points obtained in this $\lambda 7 \mathrm{~mm}$ survey have been concatenated with data points from Z09. An inspection of Figure 4 suggests that the thermal structure is stratified. The $\lambda 7 \mathrm{~mm} \mathrm{HC}_{3} \mathrm{~N}$ lines arise from a region with an excitation temperature of $7.4 \mathrm{~K}$, while the higher- $J$ transitions are from hotter regions with $T_{\mathrm{ex}}$ values from 30 to $90 \mathrm{~K}$ (Z09). The excitation temperature and the column density of $\mathrm{HC}_{3} \mathrm{~N}$ and $\mathrm{HC}_{5} \mathrm{~N}$ are consistent with the values obtained in previous works (e.g., Woods et al. 2003b). The column density of $\mathrm{HC}_{7} \mathrm{~N}$, however, is larger than the upper limit established in Fukasaku et al. (1994).

The fractional abundances of the detected molecules with respect to $\mathrm{H}_{2}$ are calculated with the equation stipulated in
Table 3

Fractional Abundances of Molecules with Respect to $\mathrm{H}_{2}$ Identified in the $\lambda 7 \mathrm{~mm}$ NRO Survey of CIT 6 and IRC+10216

\begin{tabular}{|c|c|c|c|c|}
\hline \multirow[t]{2}{*}{ Species } & \multicolumn{2}{|c|}{ CIT 6} & \multicolumn{2}{|c|}{$\mathrm{IRC}+10216$} \\
\hline & $\begin{array}{c}f_{X} \\
\left(\times 10^{-6}\right)\end{array}$ & $\begin{array}{c}\delta_{f_{X}} \\
\left( \pm \times 10^{-6}\right)\end{array}$ & $\begin{array}{c}f_{X} \\
\left(\times 10^{-6}\right)\end{array}$ & $\begin{array}{c}\delta_{f_{X}} \\
\left( \pm \times 10^{-6}\right)\end{array}$ \\
\hline $\mathrm{HC}_{3} \mathrm{~N}$ & 7 & 1 & 2.2 & 0.2 \\
\hline $\mathrm{HC}_{5} \mathrm{~N}$ & 2.6 & 0.5 & 0.8 & 0.1 \\
\hline $\mathrm{HC}_{7} \mathrm{~N}$ & 3 & 1 & 0.4 & 0.1 \\
\hline $\mathrm{SiO}$ & 0.5 & $\ldots$ & 0.3 & $\ldots$ \\
\hline $\mathrm{CS}$ & 7.0 & $\ldots$ & 2.4 & $\ldots$ \\
\hline
\end{tabular}

Olofsson (1996):

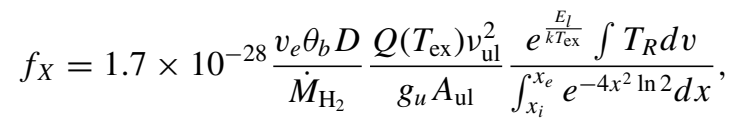

where the expansion velocity, $v_{e}$, is given in $\mathrm{km} \mathrm{s}^{-1}$, the massloss rate, $\dot{M}_{\mathrm{H}_{2}}$, in $M_{\odot} \mathrm{yr}^{-1}$, the distance $D$ in pc, the line frequency, $v_{\mathrm{ul}}$, in $\mathrm{GHz}$, and the integrated intensity, $\int T_{R} d v$, in $\mathrm{K} \mathrm{km} \mathrm{s}^{-1}$. The limits of the integration are given by $x_{i, e}=R_{i, e} /\left(\theta_{b} D\right)$, where the inner and outer boundaries of the envelopes are those from Woods et al. (2003b). The envelope boundaries of $\mathrm{HC}_{7} \mathrm{~N}$ in CIT 6 are not given by Woods et al. (2003b) but are assumed to be the same as those of $\mathrm{HC}_{3} \mathrm{~N}$ and $\mathrm{HC}_{5} \mathrm{~N}$. Other parameters are assumed to be the same as those used by Z09. The average molecular abundances are listed in Table 3, and the abundances 

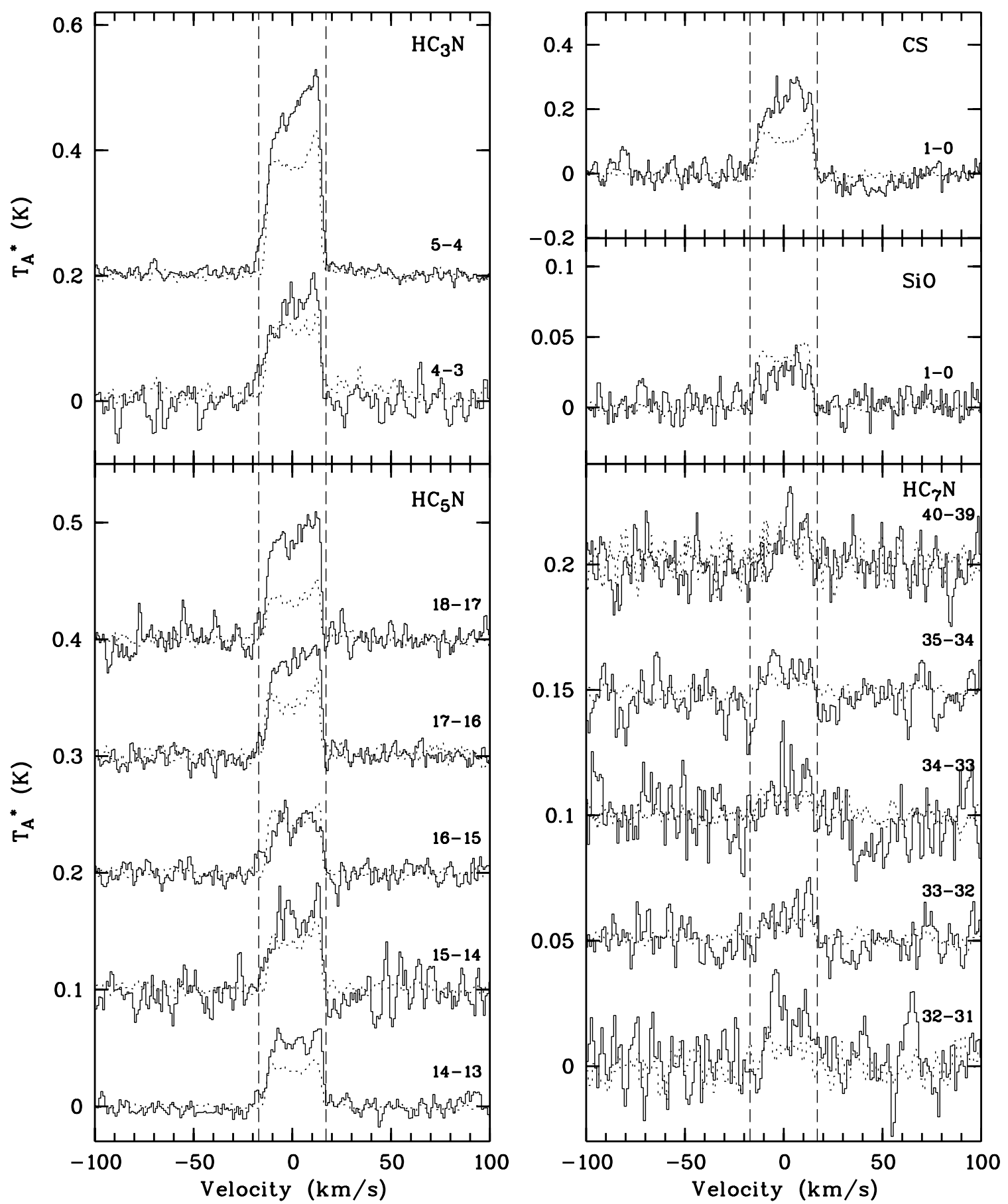

Figure 3. Profiles of the detected molecular lines in CIT 6 (solid lines) and IRC+10216 (dotted lines; scaled by a factor of 0.1).

of $\mathrm{HC}_{3} \mathrm{~N}, \mathrm{HC}_{5} \mathrm{~N}, \mathrm{SiO}$, and $\mathrm{CS}$ are in reasonable agreement with previous results. We obtain the abundance of $\mathrm{HC}_{7} \mathrm{~N}$ in CIT 6 for the first time.

\section{DISCUSSION}

\subsection{Comparison of the $\lambda 7 \mathrm{~mm}$ Spectra of CIT 6 and IRC +10216}

We compare the $\lambda 7 \mathrm{~mm}$ spectra of CIT 6 and IRC+10216 in a similar manner as in Z09. The integrated intensity ratios of the lines detected in the two objects are shown in Figure 5, where we have corrected for the beam dilution effect through multiplying the $I(\mathrm{CIT} 6) / I(\mathrm{IRC}+10216)$ line ratio by a factor of $\left(1+\theta_{b}^{2} / \theta_{\mathrm{CIT} 6}^{2}\right) /\left(1+\theta_{b}^{2} / \theta_{\mathrm{IRC}+10216}^{2}\right)$. The angular sizes of the two sources are taken to be $\theta_{\text {CIT } 6}=20^{\prime \prime}$ and $\theta_{\text {IRC }+10216}=30^{\prime \prime}$ (Fukasaku et al. 1994). Values of the correction factor range from 1.91 to 1.72 for the lines considered.

It is clear from Figure 5 that if we take the mean strength ratio of all the lines detected in CIT 6 and IRC+10216 $(\sim 0.15)$ as a standard, the $\mathrm{HC}_{n} \mathrm{~N}$ molecules are likely to be enhanced in CIT 6. As shown in Tables 2 and 3, our calculations suggest a general trend that the column densities and abundances of cyanopolyyne molecules decrease with increasing cyanopolyyne chains, suggesting that they are formed "bottomup." With stellar evolution, the central star becomes hotter and the increasing UV field can photodissociate $\mathrm{HCN}$ into $\mathrm{CN}$. 


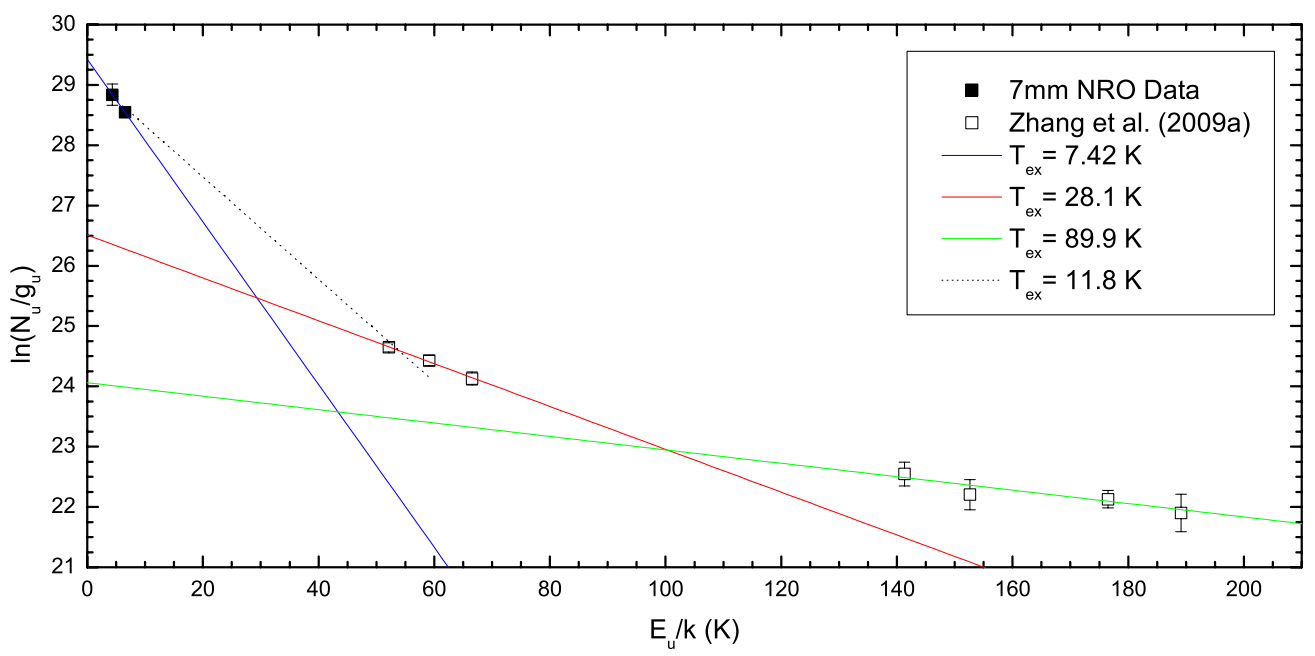

Figure 4. Rotational diagram of $\mathrm{HC}_{3} \mathrm{~N}$ in CIT 6 concatenated with data from Zhang et al. (2009a).

(A color version of this figure is available in the online journal.)

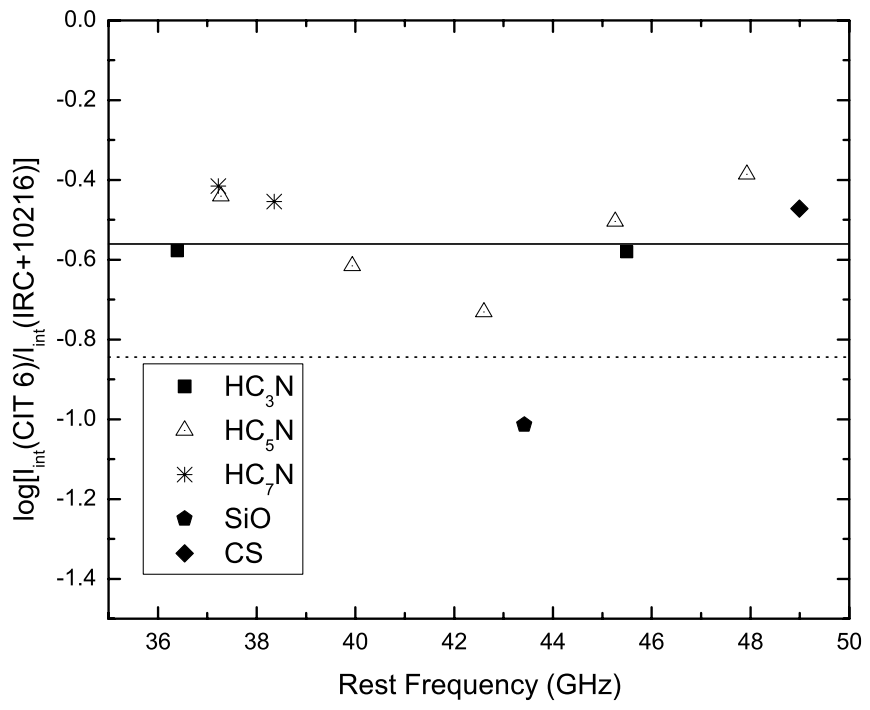

Figure 5. Integrated intensity ratios of detected molecules between CIT 6 and IRC +10216 . The effect of beam dilution has been corrected. The mean intensity ratio from this study is indicated by the solid line, while the mean value from Zhang et al. (2009a) is indicated by the dotted line.

$\mathrm{HC}_{3} \mathrm{~N}$ is then formed by $\mathrm{CN}$ and $\mathrm{C}_{2} \mathrm{H}_{2}$ via the reaction

$$
\mathrm{CN}+\mathrm{C}_{2} \mathrm{H}_{2} \longrightarrow \mathrm{HC}_{3} \mathrm{~N}+\mathrm{H} \text {. }
$$

Larger cyanopolyyne molecules are then constructed in a stepwise manner from $\mathrm{HC}_{3} \mathrm{~N}$ through

$$
\mathrm{C}_{2} \mathrm{H}+\mathrm{HC}_{2 n+1} \mathrm{~N} \longrightarrow \mathrm{HC}_{2 n+3} \mathrm{~N}+\mathrm{H}
$$

The radical $\mathrm{C}_{2} \mathrm{H}$ can be produced through the photodissociation of $\mathrm{C}_{2} \mathrm{H}_{2}$. The above reaction network is supported by the observations of Pardo et al. (2005), who compared the spectrum of IRC+10216 and that of the PPN CRL 618 and found that $\mathrm{HC}_{3} \mathrm{~N}$ and longer cyanopolyynes are rapidly enhanced by altering UV radiation field. In addition, interstellar UV photons also play an important role in cyanopolyyne chemistry. The numerical models of Woods et al. (2003a) showed that the fractional abundances of cyanopolyyne molecules increase toward the outer edge of the molecular envelope, where the interstellar UV field is stronger.
The $\mathrm{CN} / \mathrm{HCN}$ abundance ratio of CIT 6 is larger than that of IRC +10216 (Z09), suggesting that CIT 6 is likely to be more evolved and cyanopolyyne molecules are being processed. The conclusions are strengthened by the current observations of $\mathrm{HC}_{3} \mathrm{~N}, \mathrm{HC}_{5} \mathrm{~N}$, and $\mathrm{HC}_{7} \mathrm{~N}$. Our calculations show that the abundances of all the cyanopolyyne molecules in CIT 6 are larger than those in IRC +10216 , and CIT 6 has larger $\mathrm{HC}_{n} \mathrm{~N} / \mathrm{HC}_{m} \mathrm{~N}(n>m)$ ratios. Therefore, the transition from $\mathrm{HCN}$ to larger cyanopolyyne chains has occurred during the AGB stage.

$\mathrm{SiO}$ is an oxygen-bearing molecule commonly observed in C-rich stars. Its formation and depletion are related to nonLTE chemistry, shocks, and dust processes (e.g., Schöier et al. 2006; Helling \& Winters 2001). As shown in Figure 3, the I(CIT 6)/I(IRC+10216) line ratio for $\mathrm{SiO}$ behaves differently from those of cyanopolyyne molecules and is close to the $\mathrm{SiO}$ line ratio obtained in the $1.3 \mathrm{~mm}$ observations of $\mathrm{Z} 09$. This suggests that the $\mathrm{SiO}$ abundance has not been significantly altered during the evolutionary period between the two objects.

\subsection{Structure of the Envelope}

As shown in Figure 3, the lines of cyanopolyyne molecules exhibit an asymmetric profile with stronger redshifted than blueshifted emission. Such a profile can be qualitatively explained in terms of radiative transfer in an expanding optically thick envelope. In this case, the redshifted emission is mainly from the inner warmer regions, while the blueshifted emission is from the outer colder regions. In order to quantitatively calculate the line profiles, we performed one-dimensional radiative transfer modeling using the publicly available RATRAN code (Hogerheijde \& van der Tak 2000). Under the assumption of a spherically symmetric geometry, we constructed a number of models using various density, temperature, and velocity distributions, but none reproduced the observed line profiles of cyanopolyyne molecules. We find that the degree of asymmetry in the observed line profiles is far beyond the predictions of this model. Therefore, it is more likely that the asymmetric line profiles are due to non-spherically symmetric structure rather than the effect of radiative transfer. The deviation from spherical symmetry in CIT 6 can also be inferred from the polarization observations (Trammell et al. 1994; Schmidt et al. 2002). 

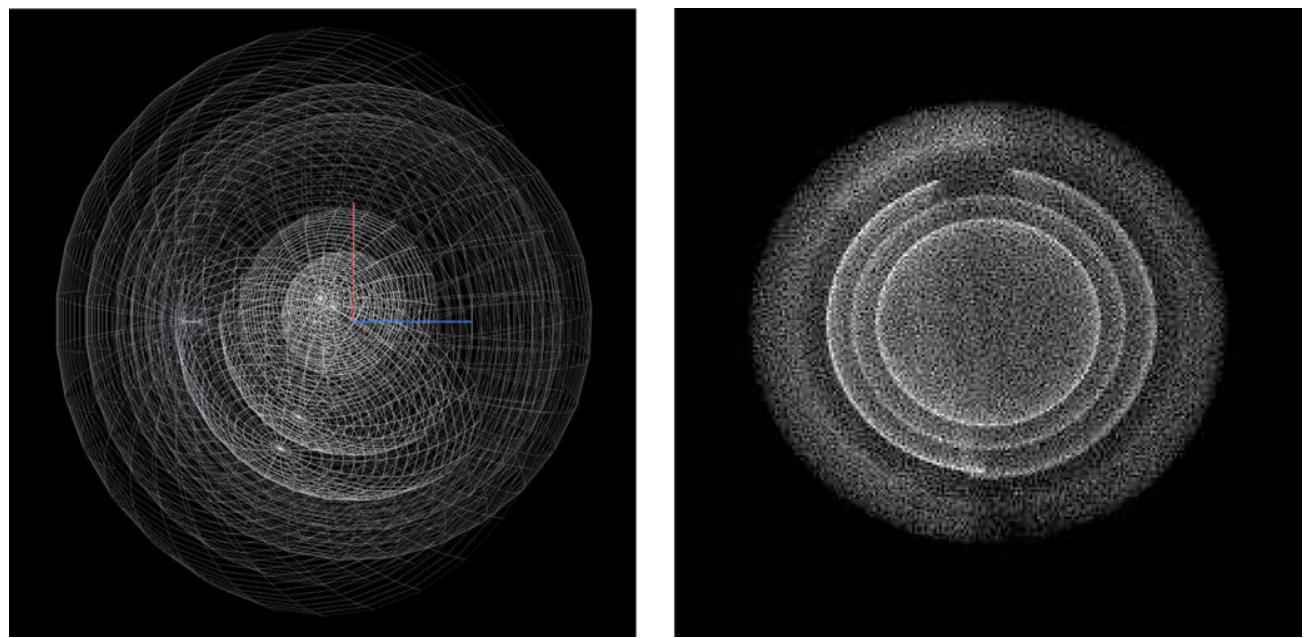

Figure 6. Shape model of CIT 6. Left panel: the mesh geometry; right panel: the rendered image. (A color version of this figure is available in the online journal.)

Furthermore, the asymmetry in the profiles of $\mathrm{HC}_{3} \mathrm{~N}$ and $\mathrm{HC}_{5} \mathrm{~N}$ lines seemed more prominent for higher- $J$ transitions. Figure 4 shows that the thermal structure is stratified and higher$J$ transitions arise from warmer regions. All of these suggest that the warmer inner regions have a more asymmetric structure.

The near-infrared (Monnier et al. 2000; Schmidt et al. 2002) and radio interferometer (Claussen et al. 2011) observations revealed that the envelope consists of several concentric arcs in different scales. Claussen et al. (2011) suggested that these arcs are the projection of some incomplete shells. Similar structure has also been observed in IRC+10216 (Dinh \& Lim 2008). Inspired by these observations, we construct a threedimensional morphokinematic model to investigate the structure of the envelope.

The modeling was performed using the Shape ${ }^{4}$ package (Steffen et al. 2011). The envelope is assumed to be optically thin and consists of 10 incomplete shells, as displayed in Figure 6. While the dimensions of each shell were specified using radial and angular extents, the orientations of the shells were assigned in terms of position angle and inclination relative to the plane of the sky. Parameters defining each shell were adjusted until the simulated line profile converged with those observed. The physical size of the shell was taken from Claussen et al. (2011). The innermost and outermost elements are, respectively, centered at a radial distance of 1".49 and 8.50, while the average separation between the shells is 0 '.78. A single Hubble law velocity distribution, with the velocity at the outer boundary defined to be $18 \mathrm{~km} \mathrm{~s}^{-1}$, is assumed. Figure 7 shows the profile of the $\mathrm{HC}_{3} \mathrm{~N}$ line reproduced by our model, which agrees well with the observations. The overall observed profile of the $\mathrm{HC}_{3} \mathrm{~N}$ line is well defined, and thus provides a good constraint on the kinematical decomposition. However, the overlying substructures of the line profiles might be complicated by spectrum noise although they can be qualitatively reproduced by our model. The observations of channel maps can provide an additional constraint in the modeling. The simulated channel maps from our model are given in Figure 8, and reproduce the observations of Claussen et al. (2011) fairly well.

These incomplete shells are presumably due to time- and space-dependent variations in mass-loss rate. However, the

\footnotetext{
4 http://www.astrosen.unam.mx/shape/
}

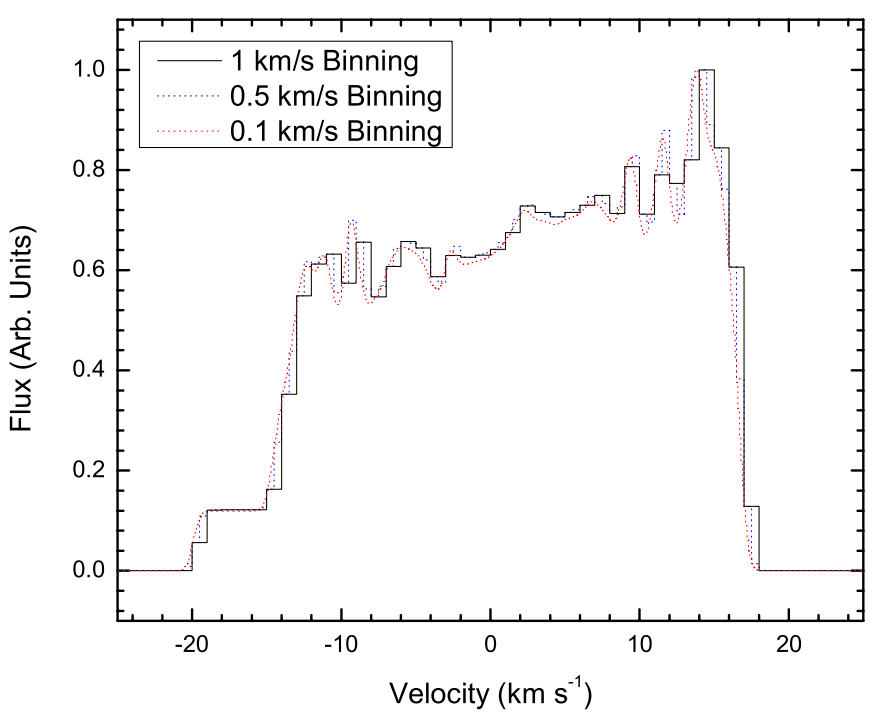

Figure 7. Profile of the $\mathrm{HC}_{3} \mathrm{~N}$ lines reproduced by the model. (A color version of this figure is available in the online journal.)

mechanism causing varying mass-loss rate is not yet well known. From the spacings between subsequent arcs observed in IRC+10216, Dinh \& Lim (2008) found that the timescale on which the mass-loss rate varies is significantly different to those of pulsation and helium flash. Soker $(1998,2000)$ showed that the magnetic activity of AGB stars can lead to semiperiodic variations in mass-loss rate. According to Soker's model, some cool regions analogous to sunspots can be formed on the surface of AGB stars where magnetic field lines exit. Dust formation is enhanced both in the cool regions and in the shadows they cast. As radiation pressure on dust grains is the mechanism of mass loss in AGB stars, the mass-loss rate can be enhanced in these cool regions. Since the magnetic activity is periodic, Soker's model can adequately account for the formation of incomplete shells. Our Shape model suggests that the time interval between two adjacent shells is about 20-30 years. This is consistent with the period of stellar magnetic activity, and thus supports Soker's model. The observed line profiles (Figure 3) suggest that the inner shells deviate more significantly from spherical symmetry than the outer shells. Since the inner shells are launched in more 


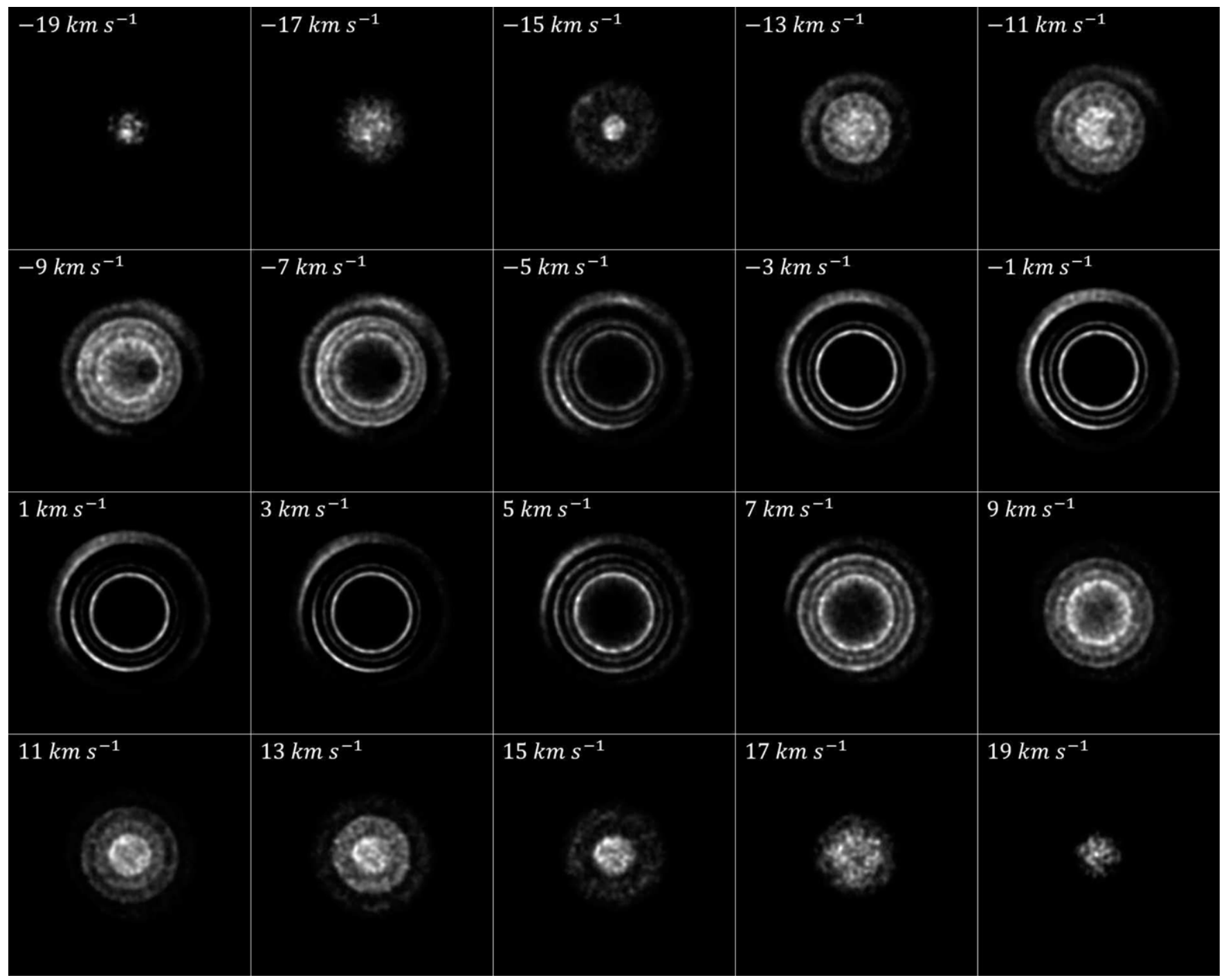

Figure 8. Channel maps reproduced by the model. Each panel is in a size of $25^{\prime \prime} \times 25^{\prime \prime}$.

recent episodes of mass loss, this implies that the asymmetry of the CSE increases with stellar evolution.

This model suggests that stellar spots might play an important role in the morphology evolution from AGB envelope to PPN and PN. The incomplete shells detached from AGB stars are probably the precursors of the rings and arcs that have been frequently observed surrounding PPNe and PNe (Hrivnak et al. 2001; Corradi et al. 2004). Furthermore, they can interact with subsequent fast stellar winds. Analogous to that of sunspots, the spatial distribution of stellar spots might have an equatorial preference, and thus the detached AGB shells confine the stellar wind into the equatorial region. It follows that the nebulae are elongated along the polar direction. Comprehensive dynamic simulations will be required to investigate this process.

\section{SUMMARY AND CONCLUSIONS}

In this molecular line survey toward CIT 6 at $\lambda 7 \mathrm{~mm}$ wavelengths, we detected a total of 25 lines belonging to $\mathrm{CS}, \mathrm{SiO}$, and several cyanopolyyne molecules. Specifically, we unambiguously detected $\mathrm{HC}_{7} \mathrm{~N}$. We determine the column densities and abundances of the detected molecules and find that longer cyanopolyyne chains are enhanced in this object compared to those in IRC +10216 . Our observations therefore provide a valuable data set to study the chemistry of cyanopolyyne molecules.

We investigate the line profiles of $\mathrm{HC}_{3} \mathrm{~N}$ and $\mathrm{HC}_{5} \mathrm{~N}$. Radiative transfer models of a spherically symmetric envelope cannot reproduce the asymmetric line profiles, suggesting that the envelope deviates from spherical symmetry. We construct a three-dimensional morphokinematic model to fit the observed line profiles as well as the channel maps obtained by Claussen et al. (2011). The results suggest that an envelope that consisted of several incomplete shells can explain these observations well. Consequently, the results of the current study may have important implications for the history of mass loss during AGB evolution.

We thank the anonymous referee for valuable comments that helped improve the paper. We also thank T. K. T. Fok, C.-H. Hsia, B. H. K. Yung, and the staff at NRO for their assistance during the observations. The work was supported by a grant awarded to Y.Z. (Project No. HKU 7073/11P), S.K. (Project No. HKU 7031/10P), and J.N. (Project No. HKU 7044/11P) from the Research Grants Council of the Hong Kong Special Administrative Region, China. 


\section{REFERENCES}

Cernicharo, J., Agúndez, M., \& Guélin, M. 2011, in IAU Symp. 280, The Molecular Universe, ed. J. Cernicharo \& R. Bachiller (Cambridge: Cambridge Univ. Press), 237

Cernicharo, J., Guélin, M., \& Kahane, C. 2000, A\&AS, 142, 181

Cernicharo, J., Waters, L. B. F. M., Decin, L., et al. 2010, A\&A, 521, L8

Claussen, M. J., Sjouwerman, L. O., Rupen, M. P., et al. 2011, ApJ, 739, L5

Cohen, M., \& Hitchon, K. 1996, AJ, 111, 962

Corradi., R. L. M., Sánchez-Blázquez, P., Mellema, G., Gianmanco, C., \& Schwarz, H. E. 2004, A\&A, 417, 637

Dinh, V. T., \& Lim, J. 2008, ApJ, 678, 303

Dinh, V. T., \& Lim, J. 2009, ApJ, 701, 292

Fukasaku, S., Hirahara, Y., Masuda, A., et al. 1994, ApJ, 437, 410

He, J. H., Dinh, V. T., Kwok, S., et al. 2008, ApJS, 177, 275

Helling, Ch., \& Winters, J. M. 2001, A\&A, 366, 229

Hogerheijde, M., \& van der Tak., F. 2000, A\&A, 362, 697

Hrivnak, B. J., Kwok, S., \& Su, K. Y. L. 2001, AJ, 121, 2775

Kawaguchi, K., Kasai, Y., Ishikawa, S.-I., \& Kaifu, N. 1995, PASJ, 47, 853

Kwok, S. 2004, Nature, 430, 26

Kwok, S. 2009, Ap\&SS, 319, 5

Lindqvist, M., Schoeier, F. L., Lucas, R., \& Olofsson, H. 2000, A\&A, 361,1036

Monnier, J. D., Tuthill, P. G., \& Danchi, W. C. 2000, ApJ, 545, 957
Olofsson, H. 1996, in IAU Symp. 178, Molecules in Astrophysics: Probes \& Processes, ed. E. van Dishoeck (Dordrecht: Kluwer), 457

Pardo, J. R., Cernicharo, J., Goicoechea, J. R., \& Goicoechea, R. 2005, ApJ, 628,275

Pardo, J. R., Cernicharo, J., Goicoechea, J. R., Guéin, M., \& Asensio, R. A. 2007, ApJ, 661, 250

Park, J. A., Cho, S.-H., Lee, C. W., \& Yang, J. 2008, AJ, 136, 2350

Patel, N. A., Young, K. H., Gottlieb, C. A., et al. 2011, ApJS, 193, 17

Schmidt, G. D., Hines, D. C., \& Swift, S. 2002, ApJ, 576, 429

Schöier, F. L., Olofsson, H., \& Lundgren, A. A. 2006, A\&A, 454, 247

Soker, N. 1998, MNRAS, 299, 1242

Soker, N. 2000, ApJ, 540, 436

Steffen, W., Koning, N., Wenger, S., Morisset, C., \& Magnor, M. 2011, IEEE Trans. Vis. Comput. Graphics, 17, 454

Tenenbaum, E. D., Dodd, J. L., Milam, S. N., Woolf, N. J., \& Ziurys, L. M. 2010, ApJS, 190, 348

Trammell, S. R., Dinerstein, H. L., \& Goodrich, R. W. 1994, AJ, 108, 984

Woods, P. M., Millar, T. J., Herbst, E., \& Zijlstra, A. A. 2003a, A\&A, 402, 189

Woods, P. M., Schöier, F. L., Nyman, L.-Å., \& Olofsson, H. 2003b, A\&A, 402, 617

Zhang, Y., Kwok, S., \& Dinh, V. T. 2008, ApJ, 678, 328

Zhang, Y., Kwok, S., \& Dinh, V. T. 2009a, ApJ, 691, 1660 (Z09)

Zhang, Y., Kwok, S., \& Nakashima, J. 2009b, ApJ, 700, 1262

Ziurys, L. M., Milam, S. N., Apponi, A. J., \& Woolf, N. J. 2007, Nature, 447, 1094 